\title{
The Design of the 3D Reconstruction System of Line Structured Light Vision
}

\author{
Tao He, Caisheng Zhu, Yulang Xie, Kexue Lai, Liangen Yang \\ Hubei Key Lab of Modern Manufacture Quality Engineering \&School of mechanical engineering \\ and Hubei University of Technology
}

Keywords: structured light; calibration; light plane; 3-d reconstruction

\begin{abstract}
This paper designs a kind of three dimensional reconstructions based on line structured light vision system. This system can realize the quick requisition of the contour point on the surface of mechanical parts, which commits precise measurement of mechanical parts. First of all, the measurement model of the system as well as a new kind of calibration method of structure light sensor had been put forward the mathematical model of the sensor calibration was also established, which only been used as a target for sensor calibration by the precision gage block with no strict requirements during calibration process of target position, which with its simple calibration solving process and easy to be used in industrial site. Second, to build the three dimensional reconstruction based on line structured light vision experiment platform. The three dimensional reconstruction software was designed, which was the system for human-computer interaction dynamic observation. Experiments show that the three dimensional reconstruction contour parts are lifelike. Users can display the three dimensional patterns of each mechanical part in all directions through the operations such as amplification, narrow, translation and rotation, which the visual effect is satisfactory.
\end{abstract}

\section{The introduction}

With the development of science and technology, the access of 3D data is becoming more and more urgent. In the process of 3D acquisition, structured light vision system is characterized by simple implementation, fast speed, low cost, non-contact, measurement etc. So the 3D coordinate measurement [2-3] based on structured light vision and target reconstruction has become one of the main directions of computer vision [1]. Structured light vision measurement was developed by laser triangulation measurement, which was widely used in the industry of visual measurement methods. Structured light vision consists of two parts, these are, structured light sensors and cameras, which are mainly used in 3D object coordinate measurement [2-3] and quality detection [4-5], etc.

\section{Solving Principle of the Contour on the Surface of Point Cloud}

Space geometric measurement model of Line structured light vision measurement, which mainly contains four coordinate system: the world coordinate system(Xw, Yw, Zw), the image plane coordinate system (x, y) and pixel coordinate system $(\mathrm{u}, \mathrm{v})$, and the camera coordinate system(Xc, $\mathrm{Yc}, \mathrm{Zc}$ ) , and the camera coordinate system axis Zc coincides with the camera itself. Distance between the two points Oc and $\mathrm{O} 1$ is an effective camera focal length $\mathrm{f}$. Coordinate transformation relation between the pixel coordinate system and world coordinate system [1]:

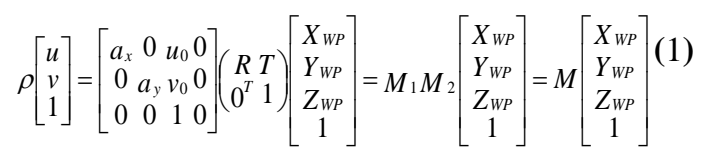

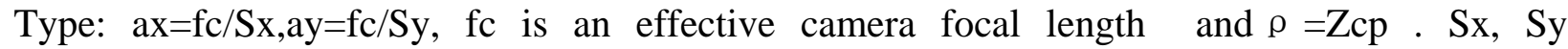
respectively are the physical size of rectangular length and width of each pixel point. (u0, v0) is pixel coordinates of camera optic axis and camera imaging plane intersection, which is also the actual center point position of image. M1 entirely decided by ax,ay,u0,v0, which has a lot to do with camera hardware structure and error of processing and manufacturing, it was also called the 
parameter matrix. M2 fully decided by $\mathrm{R}, \mathrm{T}$. R, T is parameter matrix a machine, relative to rotation and translation in the world coordinate system, which is called as matrix of the parameter. $\mathrm{M}=\mathrm{M} 1 \mathrm{M} 2, \mathrm{M}$ is $3 * 4$ matrix, which is called the projection matrix. Internal and external parameters can be identified through camera calibration technology.

Images captured by camera is a kind of transformation of the object space from 3D information to $2 \mathrm{D}$, so three-dimensional coordinate information of the object is cannot got from the simple image. From type (1) also can be seen that the type contains four unknowns, that is, $\rho$, Xwp, Ywp, Zwp, if image coordinates $(\mathrm{u}, \mathrm{v})$ is known, however ,it is unable to solve the three-dimensional coordinates $(\mathrm{Xw}, \mathrm{Yw}, \mathrm{Zw})$, thus the constraint $\mathrm{Zw}=\mathrm{f}(\mathrm{Xw}, \mathrm{Yw})$ is introduced , which is also structured light plane equation, then to find the solution to 3D space coordinate. If a line structured light plane equation for Zwp $=\mathrm{AXwp}+\mathrm{BYwp}+\mathrm{D}$ is set, then plug in type (1), the image coordinate and world coordinate mapping is got:

$$
\rho\left[\begin{array}{l}
u \\
v \\
1
\end{array}\right]=M\left[\begin{array}{c}
X_{W P} \\
Y_{W P} \\
Z_{W P} \\
1
\end{array}\right]=M\left[\begin{array}{c}
X_{W P} \\
Y_{W P} \\
A X_{W P}+B Y_{W P}+D \\
1
\end{array}\right]
$$

Tidy it:

$$
\left\{\begin{array}{l}
\rho u=m_{0} X_{W P}+m_{1} Y_{W P}+A m_{2} X_{W P}+B m_{2} Y_{W P}+D m_{2}+m_{3} \\
\rho v=m_{4} X_{W P}+m_{5} Y_{W P}+A m_{6} X_{W P}+B m_{6} Y_{W P}+D m_{6}+m_{7} \\
\rho=m_{8} X_{W P}+m_{9} Y_{W P}+A m_{10} X_{W P}+B m_{10} Y_{W P}+D m_{10}+m_{11}
\end{array}\right.
$$

Then got:

$$
\left\{\begin{aligned}
X_{W P} & =\frac{F J-G I}{E F-H I} \\
Y_{W P} & =\frac{J H-E G}{E F-H I} \\
Z_{W P} & =A X_{W P}+B Y_{W P}+D
\end{aligned}\right.
$$

In type:

$$
\begin{aligned}
& E=m_{0}+A m_{2}-u m_{8}-A u m_{10} \\
& F=v m_{9}+B v m_{10}-m_{5}-B m_{6} \\
& G=D v m_{10}+v m_{11}-D m_{6}-m_{7} \\
& H=m_{4}+A m_{6}-v m_{8}-A v m_{10} \\
& I=u m_{9}+B u m_{10}-m_{1}-B m_{2} \\
& J=\text { Dum }_{10}+u m_{11}-D m_{2}-m_{3}
\end{aligned}
$$

At this point, model of line structured light measurement system has been completed, any measured contour point in the image plane coordinate system of the spatial coordinates can be solved by equations (4).

\section{the Camera Calibration}

MVtec's image processing software HALCON from Germany has achieved good results in practical application in camera calibration, with its higher calibration precision, high operation efficiency, good cross-platform portability and simple operation. As shown in figure 1 is HALCON camera, the calibration board specifications in this experiment are: calibration circular array $7 * 7$, calibration circle diameter $4 \mathrm{~mm}$, distance between adjacent calibration circle center is $8 \mathrm{~mm}$, 
external border $64 \mathrm{~mm}$, length of internal border $60 \mathrm{~mm}$.

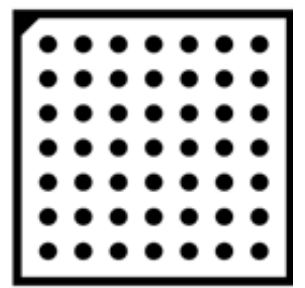

Figure 1.Calibration plate of HALCON

Calibration precision of HALCON camera relates with the number of plate image and position of calibration plate. As shown in the figure, different locations within the scope of the camera view to obtain images of calibration plate. This experiment took 25 calibration plate images, making the calibration board cover all the camera sight, on account of different distortion of camera lens on different position of image coordinates. Internal and external parameters of calibration of camera using Halcon image processing software have been shown in the table below:

Table 1.Camera internal calibration results

\begin{tabular}{|c|c|c|c|c|c|c|c|}
\hline & $(\mathrm{um})$ & $(\mu \mathrm{m})$ & $\mathrm{F}_{\mathrm{d}}(\mathrm{mm})$ & $(1 / \mathrm{m}$ & Cxp(pixels) & Cy(pixels) & RMS erro(pixelsts) \\
\hline Call & 5.20292 & 5.2 & 16.4076 & 36 & 611.528 & 510.185 & \\
\hline
\end{tabular}

Table 2. external calibration results of the camera

\begin{tabular}{|c|c|c|c|c|c|c|}
\hline parameter & $\begin{array}{c}\text { Position and } \\
\text { posture on } \\
\text { direction } \mathrm{X}(\mathrm{m} \\
\mathrm{m})\end{array}$ & $\begin{array}{c}\text { Position and } \\
\text { posture on } \\
\text { direction } \mathrm{Y}(\mathrm{m} \\
\mathrm{m})\end{array}$ & $\begin{array}{c}\text { Position and } \\
\text { posture on } \\
\text { directionZ(m } \\
\mathrm{m})\end{array}$ & $\begin{array}{c}\text { Rotation on } \\
\mathrm{X} \text { direction } \\
\text { (c) }\end{array}$ & $\begin{array}{c}\text { Rotation on } \\
\mathrm{Y} \text { direction } \\
\text { (c) }\end{array}$ & $\begin{array}{c}\text { Rotation on } \\
\mathrm{Z} \text { direction } \\
\text { (c) }\end{array}$ \\
\hline $\begin{array}{c}\text { Calibration } \\
\text { results }\end{array}$ & 12.965 & 2.80822 & 424.852 & 1.34269 & 359.874 & 18.8596 \\
\hline
\end{tabular}

Select a calibration plate image among 25 images as a reference frame in the HALCON software (reference frame is the world coordinate system in this experiment), demarcating the external parameter of the camera. the reference image selected in this experiment calibration plate is images on the coordinate plate placed on the measuring platform, making the plane of world coordinate system $\mathrm{Zw}=0$ coincides with precision measurement platform, then calculating the coordinate $\mathrm{Zw}=0$ is the size of the measured object. Calibration results in the following table:

\section{structured light plane calibration}

P1, P2, P3 are the points on the structured light stripe, which P1, P2, P3 in ZW= 0 plane, P2 in the plane with the height $\mathrm{ZW}=\mathrm{h}$. Known by the geometry: three collinear points can determine a plane, thus by P1, P2, P3 can determine the structure parameters of the light plane. Because coordinates of $\mathrm{Zw}$ are known XW, YW coordinates can be calculated by (1) type, light plane equation can be solved by the following type:

$$
\left|\begin{array}{ccc}
X-X_{W 1} & Y-Y_{W 1} & Z-Z_{W 1} \\
X_{W 2}-X_{W 1} & Y_{W 2}-Y_{W 1} & Z_{W 2}-Z_{W 1} \\
X_{W 3}-X_{W 1} & Y_{W 3}-Y_{W 1} & Z_{W 3}-Z_{W 1}
\end{array}\right|=0 \text { (5) }
$$

In actual process of calibration, error is bigger when using the method of 3 points determine a plane .Generally, method of multi-points to fit a plane is used. Concrete method is: gage blocks (level 0) of high precision with different height $10 \mathrm{~mm} / 40 \mathrm{~mm} / 70 \mathrm{~mm}$ placed on the precision measurement platform, in order to reduce the calibration error and ensure the combining of gage blocks with precision measuring platform, take the image of structured stripe on the surface of the different height of gauge block, and then overlay image by image. Laser stripe images are the images with different heights after image overlay. Take points on laser stripe of different height; bring each coordinates of each point in type (1) for calculating the corresponding world coordinates. Work out world coordinates of each point and make use of the least squares method to fit the plane, then obtain the structural parameter of the plane. The parameters in the chart below is the image 
coordinates and corresponding spatial coordinates of the selected point in this experiment:

Table 3.Data used in the coordinate points in structured light plane calibration

\begin{tabular}{|l|l|l|l|l|}
\hline \multicolumn{2}{|l|}{ Pixel coordinates } & \multicolumn{3}{l|}{ world coordinates } \\
\hline $\mathrm{u}($ Pixel $)$ & $\mathrm{v}($ Pixel ) & $\mathrm{Xw}(\mathrm{mm})$ & $\mathrm{Yw}(\mathrm{mm})$ & $\mathrm{Zw}(\mathrm{mm})$ \\
\hline 260 & 401.1471 & -61.5254 & 2.6893 & -10 \\
\hline 875 & 535.3818 & 20.5257 & -6.7319 & -10 \\
\hline 645 & 760.4097 & 0.1835 & 28.2687 & -40 \\
\hline 190 & 661.7299 & -56.2197 & 34.7569 & -40 \\
\hline 230 & 994.2048 & -36.8651 & 65.3877 & -70 \\
\hline 480 & 1048.3308 & -8.2878 & 62.1291 & -70 \\
\hline
\end{tabular}

Fit the plane by the least squares method using World coordinates in the above table , finally structural parameter of structured light plane is concluded, light plane equation is: $\mathrm{Zw}=-$ $0.104969 \mathrm{Xw}-0.915447 \mathrm{Yw}-14.032457$, world coordinates of all points of structured light stripe can be solved after bring them in the type .

\section{The design of 3D reconstruction system}

Hardware system design

Hardware system connection as shown in figure 2, its measuring principle and the process is: main controlling computer sending PLC control word to logic controller, transistor PLC port highspeed pulsing to stepping drive, a stepper motor driving screw rotation driving, making the object set on one dimensional motion platform move like translational motion under structured light, the grating ruler will move speed and displacement information feedback to the computer, the computer control the cameras collecting stripe images of structured light isometric ally. collected stripe images were processed for the real-time image by computer, stripes feature points were extracted, then plus them in the model of line structured light vision, three-dimensional space coordinates of the object can be solved.

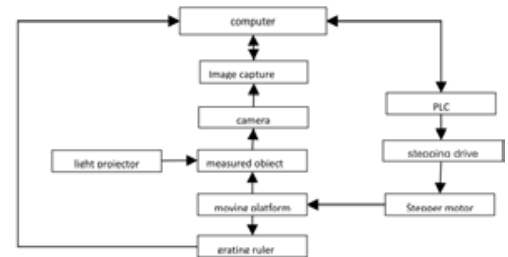

Figure 2.Hardware connection diagram of line vision measurement system

As shown in figure 3, experimental platform of the line structured light vision measurement system was designed by figure 2. The hardware part mainly consists of USB camera, projector of the line structured light, stepper motor, one dimensional mobile platform, computer, PLC; the angle of preliminary design between camera optic axis and light plane is about $20^{\circ}$, the cameras installed vertically, camera field of vision $150 \mathrm{~mm} * 200 \mathrm{~mm}$, and the system measuring depth was from 0 $\mathrm{mm}$ to $80 \mathrm{~mm}$.

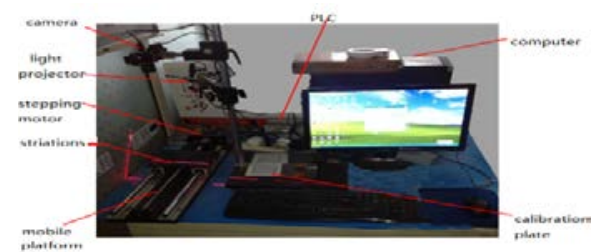

Figure 3. experimental platform of line structured light vision measurement 
The main hardware parameters as shown in table 3.

Table 3 hardware parameters of Linear structured light measurement system

\begin{tabular}{|c|c|c|c|}
\hline name & model & \multicolumn{2}{|c|}{ The main parameter index } \\
\hline \multirow{3}{*}{ Camera CCD } & \multirow{3}{*}{$\begin{array}{l}\text { DCam } 130 \mathrm{~m} \\
\text { black and white }\end{array}$} & resolution & $1024 * 1280$ \\
\hline & & Pixel size $(\mu \mathrm{m})$ & $5.2 * 5.2$ \\
\hline & & $\begin{array}{l}\text { Data } \\
\text { transmission } \\
\text { interface }\end{array}$ & USB \\
\hline $\begin{array}{l}\text { Structured light } \\
\text { sensor }\end{array}$ & $\begin{array}{l}\text { YD-L650P50- } \\
18\end{array}$ & $\begin{array}{l}\text { The output wave } \\
\text { length }\end{array}$ & $650 \mathrm{~nm}$ \\
\hline The lens & computar & The focal length & $16 \mathrm{~mm}$ \\
\hline
\end{tabular}

\section{Software system design}

Software part is mainly responsible for sending control characters to the PLC, making mobile platform move under effective control, including the control of movement speed and displacement of the mobile platform and so on, then making acquisition and post processing of image information of the object to be measured, coordinate to be solved, finally realizing the measurement purposes.

Main functions of Software system are: accurate control of the speed and displacement of one dimensional motion platform, the real-time image of the stripe of line structured light to be captured accurately and rapidly and saved, preprocessing the image, then extracting the center of the line structured light stripe, establishing a friendly man-machine interface, finally realizing the manmachine interactive dynamic observation of the model. Software architecture consists of three modules, the software system is based on the development of VS2010 platform. Main purposes of three modules of software architecture:

Calibration module: implementation of the calibration to camera and light plane. Calibration to camera by HALCON, using open-source code Opencv to preprocess and extract stripe image of structured light, to extract the coordinates of feature points, and then realize the calibration of the light plane.

System measurement module: collection of stripe image of the line structured light, communication with PLC and grating ruler, control motor movement, extract stripe feature points of structured light, then to make coordinate transformation.

Display saving:save the coordinates of surface contour of the object to be measured, establish three-dimensional model on the surface of the object under test using OpenGL , and achieve a friendly man-machine interactive operation, dynamic rotation, amplification, narrowing, and translation operation.

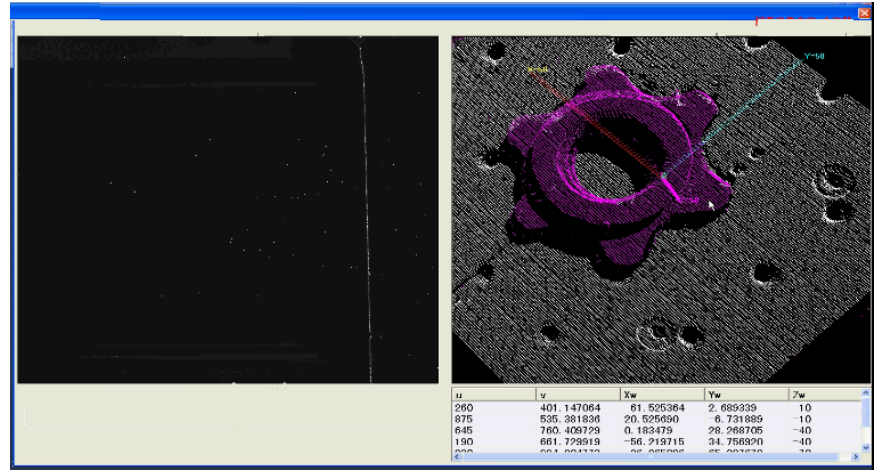

Figure 4. software interface 


\section{Conclusion}

First of all, experiment of camera calibration was done. Realizing precision of camera calibration by using HALCON, accurate calibration on the light plane again, and then designed and manufactured the experiment device of the line structured light vision measurement system and developed the software testing system. Through saving the coordinates on the surface of the parts to be measured, establish a three-dimensional model using OpenGL on the surface of the parts to be tested.

\section{Acknowledgments}

This paper is supported by Natural Science Foundation of China (No.51275158, No.51275157), and supported by Natural Science Foundation of Hubei Province (No.2012FFA056).

\section{References}

[1] Graebling P, Aliment A, Zhou D, et al. Optical high precision three-dimensional vision-based quality control of manufactured parts by use of synthetic images and knowledge for image-data evaluation and interpretation[J].Applied Optics,2002,(41):2627-2613.

[2] Jigui Zhu, Zhijing Yu, visual measuring principle and the method [M]. Beijing: China Machine Press, 2011.4

[3] Nick Van Gestel, Steven Cuypers, Philip Bleys. A performance evaluation test for laser linear scanners on CMMs [J].Optics and Lasers in Engineering, 2009, (47):336-342.

[4] Wu B, Xue T, Zhang T, et al .A novel method for round steel measurement with a multi-linear structured light vision sensor[J]. Measurement Science and Technology, 2010,21(2):025204025209.

[5] Zhang G, He J, Li X.3D vision inspection for internal surface based on circle structured-light [J].Sensors and Actuators A: Physical, 2005, 122(1):68-75. 